\title{
FABRICATION AND TESTING OF A HIGH RESOLUTION EXTENSOMETER BASED ON RESONANT MEMS STRAIN SENSORS
}

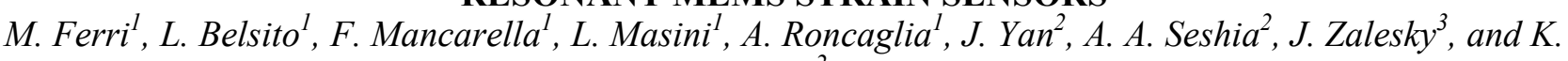 \\ $\operatorname{Sog} a^{2}$ \\ ${ }^{1}$ Institute of Microelectronics and Microsystems, CNR, Bologna, Italy \\ ${ }^{2}$ Department of Engineering, University of Cambridge, Cambridge, United Kingdom \\ ${ }^{3}$ Czech Technical University in Prague, Faculty of Civil Engineering, Prague, Czech Republic
}

\begin{abstract}
A novel type of linear extensometer with exceptionally high resolution of $4 \mathrm{~nm}$ based on MEMS resonant strain sensors bonded on steel and operating in a vacuum package is presented. The tool is implemented by means of a steel thin bar that can be pre-stressed in tension within two fixing anchors. The extension of the bar is detected by using two vacuum-packaged resonant MEMS double- ended tuning fork (DETF) sensors bonded on the bar with epoxy glue, one of which is utilized for temperature compensation. Both sensors are driven by a closed loop self-oscillating transresistance amplifier feedback scheme implemented on a PCB (Printed Circuit Board). On the same board, a microcontroller-based frequency measurement circuit is also implemented, which is able to count the square wave fronts of the MEMS oscillator output with a resolution of $20 \mathrm{nsec}$. The system provides a frequency noise of $0.2 \mathrm{~Hz}$ corresponding to an extension resolution of $4 \mathrm{~nm}$ for the extensometer. Nearly perfect temperature compensation of the frequency output is achieved in the temperature range $20-35^{\circ} \mathrm{C}$ using the reference sensor.
\end{abstract}

\section{KEYWORDS}

Extensometer, MEMS resonator, strain sensor, vacuum packaging

\section{INTRODUCTION}

The use of MEMS (Micro Electro Mechanical Systems) resonant strain sensors for direct measurement of structural strain on materials like steel and aluminum has been recently proposed as a candidate technology for ultra-high resolution strain detection [1]. Thanks to their outstanding performance, such sensors show high potential for many applications, most of which are still unexplored at present.

In this paper, MEMS resonant strain sensors bonded on steel and packaged in vacuum are utilized for demonstrating a novel type of linear extensometer with exceptionally high resolution.

\section{DESIGN AND FABRICATION}

\section{Mechanical design}

In Fig. 1, a picture of the MEMS based extensometer prototype is reported. The tool is realized with a roughly $20 \mathrm{~cm}$ long and $0.2 \mathrm{~mm}$ thick steel bar onto which a resonant MEMS strain sensor of the double-ended tuning fork (DETF) configuration is bonded with epoxy glue (TorrSeal from Varian) and packaged in vacuum using the technique described in [2]. The steel bar is designed according to the dimensions reported in Fig. 2 and connected at its extremes at two anchors fabricated with thicker steel that are used to fix the extensometer to the structure of interest.

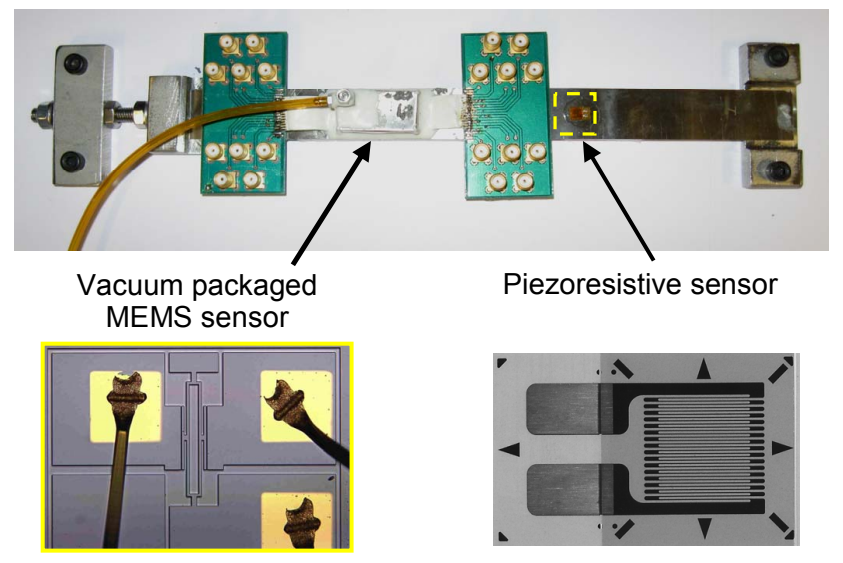

Figure 1: Prototype of extensometer based on resonant MEMS strain sensors operating in vacuum.

On the bar, a commercial piezoresistive strain sensor (CEA-13-250UN-120 strain gauge from Vishay, visible on Fig. 1 on the right) is also fixed on the side of the box containing the two MEMS sensors, in order to be used as a reference in strain calibration of the extensometer.
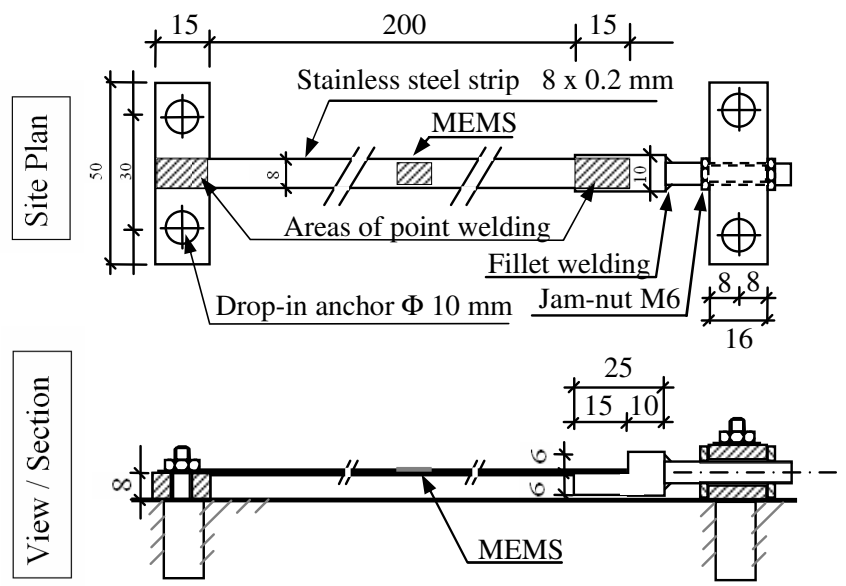

Figure 2: Mechanical design of the extensometer: top view (top) and cross-section (bottom), dimensions in mm.

Both the MEMS devices employed are packaged in vacuum within a steel box fixed on the bar by vacuum glue (Torr Seal from Varian) and connected to the driving 
circuitry on PCB by SMA cables, using two sets of connectors placed on both sides of the package (see Fig. $1)$.

\section{MEMS sensor design and fabrication}

The DETF sensor used in the extensometer was fabricated using a thick SOI technology $(15 \mu \mathrm{m}$ thick device layer) reported earlier [3]. A micrograph showing the geometry of the MEMS device employed is shown in Fig. 3.

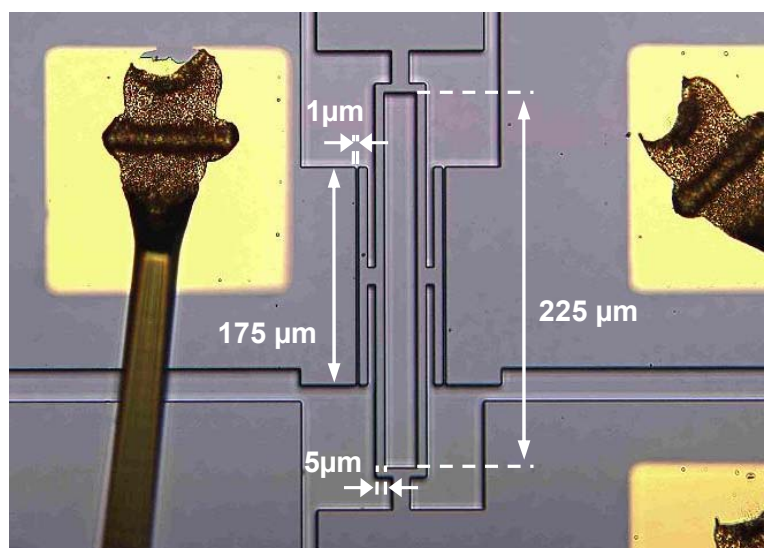

Figure 3: MEMS strain sensor geometry used in the extensometer.

The two prototypes employed in the extensometer were designed according to the dimensions shown in Fig. 2, with tine length of $225 \mu \mathrm{m}$, tine width of $5 \mu \mathrm{m}$, actuation electrode width of $175 \mu \mathrm{m}$, and coupling gap of approximately $1 \mu \mathrm{m}$. Both devices were wire bonded to the external PCB driving electronics through $\mathrm{TiN} / \mathrm{Au}$ bonding pads (also visible in the figure).

The sensing and reference MEMS were both operated in a closed loop configuration with a DC bias of around $30 \mathrm{~V}$, according to the circuit scheme described below.

\section{Implementation of read-out electronics on PCB}

For the closed loop operation of the MEMS sensor used in the extensometer and the frequency shift detection, a prototype PCB circuit was designed and implemented. In the circuit, two identical closed loop transresistance amplifier oscillation schemes were implemented, in order to drive two MEMS sensors at the same time and use one of them as a reference for temperature drift compensation. The reference sensor was placed on the same package of the strain sensing device but oriented in transverse direction with respect to the extensometer axis, in order to be insensitive to axial strain.

The feedback loop used to operate the resonant MEMS sensors in self-oscillation mode was designed according to the transresistance amplifier scheme, including a comparator for conversion of the oscillation signal into a square wave and a band pass filter used to reduce the noise [4], as illustrated in Fig. 4.

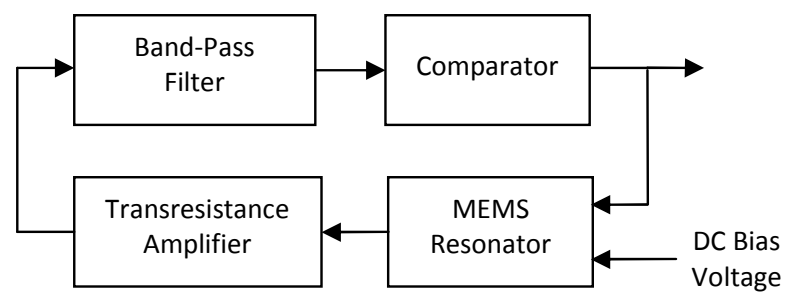

Figure 4: Feedback scheme used for closed-loop operation of the MEMS resonant sensors in the extensometer.

On the PCB (Fig. 5), a microcontroller-based frequency counting circuit was also implemented, designed to work by counting the square wave fronts of the MEMS oscillators output on a counting time of roughly $150 \mathrm{msec}$, precisely measured by sampling the first and last front of the observed waveform with a resolution of $20 \mathrm{nsec}$, and deriving the frequency from the number of counted fronts divided by the measured interval.
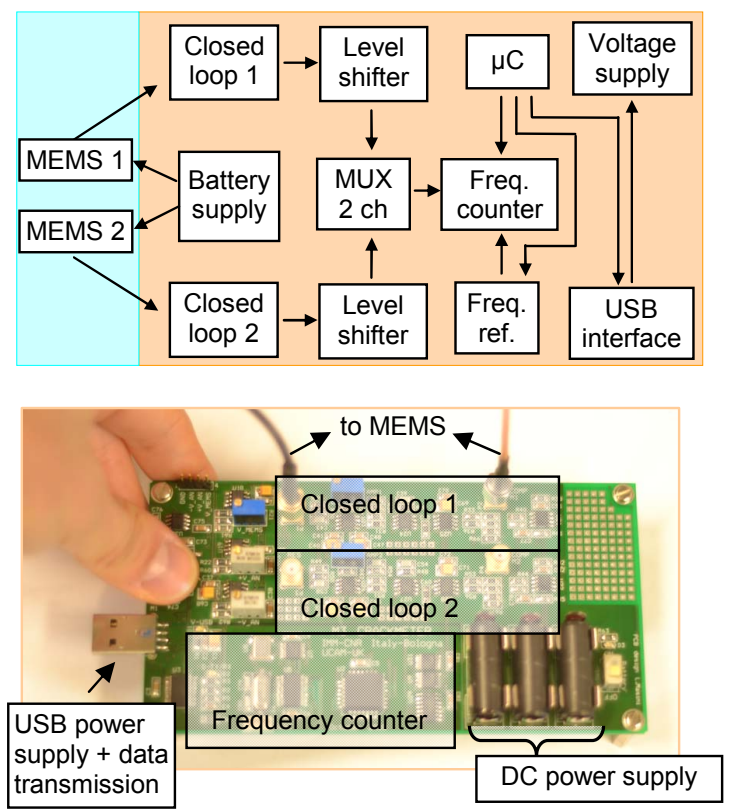

Figure 5: Extensometer readout board implementing two independent closed loop driving circuits and a microcontroller-based digital frequency counter.

\section{TESTING}

The strain sensitivity of the device was determined by applying tension to the extensometer by means of a pre-stressing screw (visible in Fig. 1 on the left of the tool) once the mechanical anchors are fixed. The tensile strain produced on the steel bar was measured by means of the reference piezoresistive strain sensor and calibrated against the frequency shift of the resonator peak, measured in open loop (Fig. 6). The measured frequency 
shift-strain calibration curve in the strain range 15-120 $\mu \varepsilon$ was fairly linear with an average sensitivity around 10 $\mathrm{Hz} / \mu \varepsilon$.

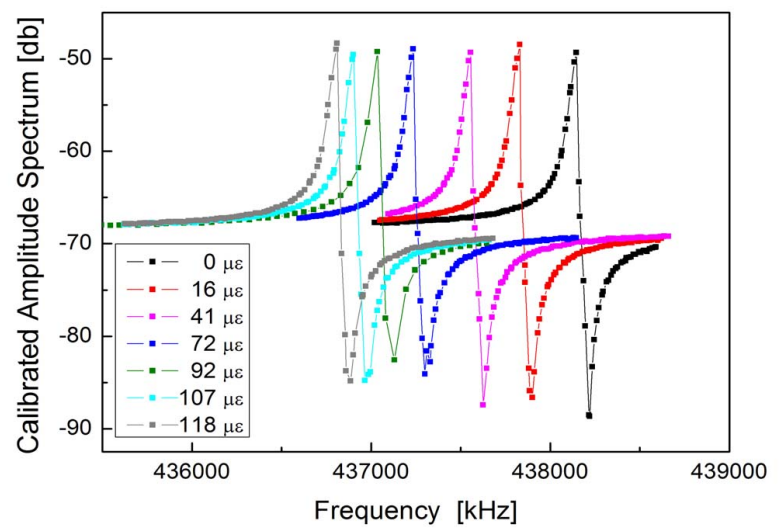

Figure 6: Strain-frequency shift calibration of the MEMS sensor (3 mTorr vacuum level), showing a strain sensitivity around $10 \mathrm{~Hz} / \mu$.

In order to evaluate the noise floor of the MEMS oscillator, which determines the resolution of the sensor in terms of strain and, consequently, the resolution of the extensometer in terms of extension, the short-term frequency jitter of the frequency counter was measured without applied strain and maintaining a constant temperature in the environment during the experiment. From this measurement, a frequency noise around $0.2 \mathrm{~Hz}$ on a $10 \mathrm{sec}$ averaging time was estimated, as shown in Fig. 7.

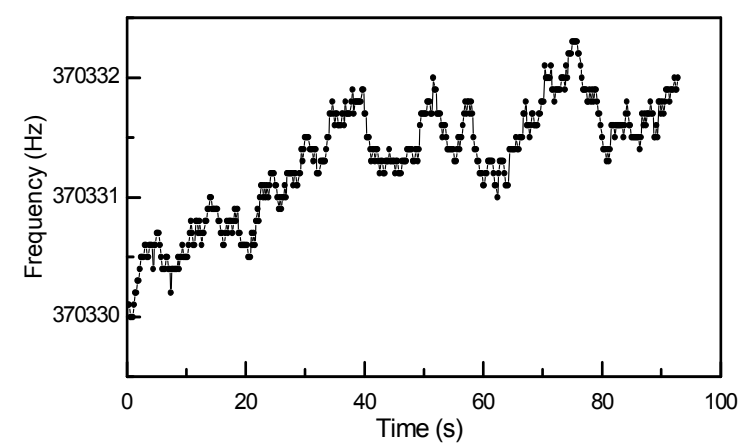

Figure 7: Short-term frequency stability on MEMS frequency output with roughly $0.2 \mathrm{~Hz}$ maximum jitter.

From the measurements of frequency noise and the strain sensitivity, a strain resolution of $20 \mathrm{n} \varepsilon$ for the MEMS sensor (used in the extensometer) can be derived, which corresponds to an exceptionally high extension resolution of $4 \mathrm{~nm}$ on the $20 \mathrm{~cm}$ length of the tool.

In Fig. 8, the performance of the temperature drift compensation scheme using the reference sensor is reported, showing the plots of the two MEMS oscillator frequency values acquired simultaneously and their difference while the sensor package was artificially heated and cooled with air jets. As may be seen, the use of the reference sensor permits almost perfect compensation of temperature drift of the sensor output. The temperature range considered in the experiment was roughly 20-35 ${ }^{\circ} \mathrm{C}$.

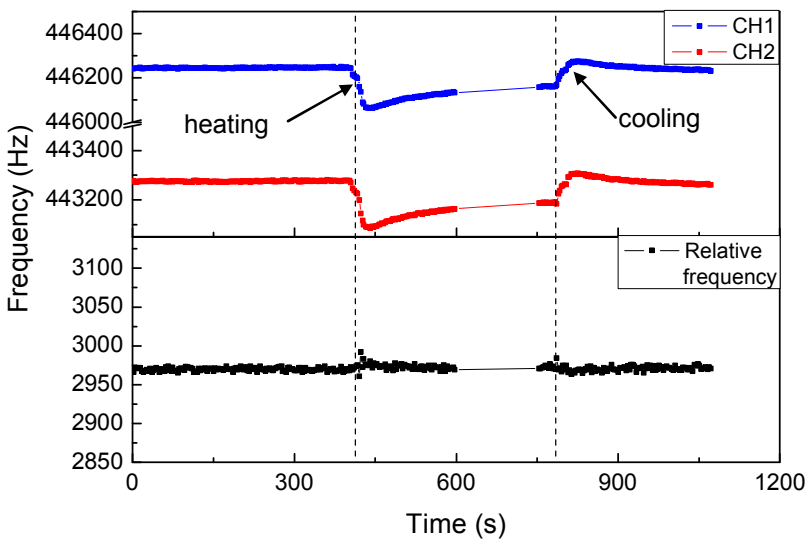

Figure 8: Temperature drift compensation scheme based on in-package MEMS reference resonator.

\section{CONCLUSIONS}

A novel type of linear extensometer based on resonant MEMS strain sensors operated in vacuum has been presented. The extensometer was manufactured with a thin steel bar onto which the MEMS sensors were bonded and packaged. The devices were operated in self oscillation mode by means of PCB driving electronics and the frequency shift of the MEMS resonator induced by mechanical strain on the tool was measured by a microcontroller-based frequency measurement circuit, also implemented on the PCB.

Due to the low short-term frequency noise of approximately $0.2 \mathrm{~Hz}$, the MEMS extensometer showed an exceptionally high resolution of $4 \mathrm{~nm}$ in terms of extension measurement. Moreover, the use of a reference MEMS sensor placed along the transverse direction of the extensometer allowed for almost perfect compensation of the sensing signal temperature drift in the range $20-35^{\circ} \mathrm{C}$.

\section{REFERENCES}

[1] A. P. Pisano, "MEMS and Nanotechnology for the Handheld, Portable Electronic and the Automotive Markets”, in Digest Tech. Papers Transducers'07 Conference, Lyon, June 10-14, 2007, pp. 1-4.

[2] M. Ferri, S. Cristiani, Y. Kobayashi, K. Soga and A. Roncaglia, "A packaging technique for silicon MEMS strain sensors on steel", Proceedings of IEEE Sensors 2008, Lecce, Oct. 26-29, pp. 1524-1527.

[3] M. Ferri, F. Mancarella, L. Belsito, A. Roncaglia, J. Yan, A. A. Seshia, K. Soga, J. Zalesky, "Strain sensing on steel surfaces using vacuum packaged MEMS resonators", Proc. Eurosensors XXIV, September 5-8, 2010, Linz, Austria.

[4] J. E-Y. Lee, B. Bahreyni, Y. Zhu and A. A. Seshia, A 
Single-Crystal-Silicon Bulk-Acoustic-Mode Microresonator Oscillator, IEEE Electron Device Letters, Vol. 29, No. 7, pp. 701-703, July 2008.
CONTACT

* M. Ferri, tel: +39-051-6399121; ferri@bo.imm.cnr.it 